\title{
Competencias y subcompetencias para \\ la atención dietoterapéutica del sobrepeso \\ y la obesidad infanto juvenil, en la formación de nutricionistas
}

\section{Competences and subcompetences in the nutricionist formation for the diet therapeutic assistance of overweight and obesity of child and adolescent population}

\section{ABSTRACT}

The aim of this study was to identify the competences and subcompetences required for providing diet therapy to children and adolescents with overweight and obesity which must be developed during the training of nutritionists. In order to identify them we utilized the advice of an expert in the subject. A semistructured survey using the technique of personal interview was applied to 16 nutritionists from several labor fields that were in charge of the diet therapy of overweight and obese children and adolescents. With the information obtained, a list of competences was elaborated which were then analyzed and categorized by a team of academics in order to define the respective sub-competences. We conclude that it is possible to identify competencies and sub-competences required by nutritionists to provide diet therapy attention to children and adolescent with overweight and obesity.

Key words: competences, sub-competences, training of nutritionists, child and adolescent overweight and obesity.

\section{INTRODUCCIÓN}

El sobrepeso y la obesidad constituyen un importante problema de salud pública que afecta a la toda la población en diversas partes del mundo. La obesidad es considerada factor de riesgo para varias enfermedades crónicas en adultos y en la población infanto juvenil $(1,2)$.

Al respecto, el Ministerio de Salud de Chile ha desarrollado múltiples estrategias para detener la incidencia de esta epidemia y disminuir su prevalencia $(3,4)$. Dentro de estas está la Estrategia Global contra la Obesidad (EGO), la que presenta como pilares fundamentales, la alimentación saludable y la actividad física (4).

La alimentación como un proceso humano complejo, requiere contar con recursos humanos competentes, que permitan desarrollar un modelo de la alimentación saludable. Dentro de este enfoque se encuentra la dietoterapia necesaria de ser aplicada en las personas afectadas por sobrepeso u obesidad. Al respecto, uno de los recursos humanos requerido para aplicar los tratamientos dietéticos en cada caso, es el
Ana María Labraña T

Eliana Durán F.

Delia Soto Á.

Departamento de Nutrición y Dietética, Facultad de Farmacia, Universidad de Concepción, Concepción, Chile.

Dirigir correspondencia a:

Profesora Ana María Labraña Torres
Departamento de Nutrición y Dietética Facultad de Farmacia Universidad de Concepción Casilla 237 Concepción, Chile

Teléfono: 52-41-2726510

E-mail: alabrana@udec.cl

Este trabajo fue recibido el 31 de Diciembre de 2014 y aceptado para ser publicado el 10 de Mayo de 2015. nutricionista, profesional que en Chile es considerado como recurso básico de acción en los diferentes programas de salud de las personas $(5,6)$.

Por lo anterior, la formación académica de este profesional debe considerar las competencias específicas y genéricas que le permitan un desempeño al más alto nivel en escenarios reales y complejos. En este sentido la educación superior actualmente ha desarrollado programas de mejoramiento institucional para el pregrado, para lo cual se han definido estrategias de innovación curricular, las que contemplan como eje principal, el aprendizaje basado en competencias cuya definición plantea que el estudiante adquiera la capacidad para poner en práctica conocimientos de manera integrada y autónoma, habilidades, actitudes y valores para enfrentar y poder resolver problemas reales (7-10).

Para operacionalizar el desarrollo de competencias se requiere de la formulación de subcompetencias, de las cuales derivan los resultados de aprendizaje (RA), los que deben estar presentes en cada programa de asignatura. Esto permite 
cautelar el logro de los aprendizajes en determinados niveles de profundidad, según la etapa del proceso formativo. Por otra parte la innovación curricular implica el desarrollo de una serie de etapas extensas y secuenciales partiendo por la definición de las competencias profesionales para continuar con las competencias del egresado $(7,9)$.

El objetivo de este trabajo fue identificar las competencias y las subcompetencias requeridas por los nutricionistas, para brindar la atención dietoterapéutica a población infanto juvenil con sobrepeso y la obesidad.

\section{SUJETOS Y MÉTODOS}

Para identificar las competencias, se contó con la asesoría de una experta en el tema, quien a partir del un Manual Metodológico elaborado para tal efecto por la OPS/OMS el año 1990 (11), adaptó una encuesta semi estructurada, para ser aplicada mediante técnica de entrevista personal, a 16 nutricionistas provenientes de diversos campos laborales, quienes registraron las competencias que a su juicio debiera tener el nutricionista en la atención dietoterapéutica del sobrepeso y la obesidad infanto juvenil.

La encuesta fue previamente validada mediante juicio de expertos $(11,12)$, y contempló el nivel de importancia de la competencia, con la siguiente clasificación: muy impor- tante, siempre la utilizo, el pregrado contribuyó totalmente a lograrla. Cada una de estas debía ser calificada en la escala de 1 a 4 , siendo el 1 el puntaje mínimo y 4 el máximo. Para el análisis se consideraron las respuestas cuyas competencias fueron calificadas con puntaje 4 (máximo) correspondiente al estándar o criterio de calificación esperado. Con la información obtenida se elaboró un listado de competencias las que posteriormente a través de un taller integrado por nutricionistas académicas, se refundieron y se clasificaron en específicas y generales. Una vez analizadas y categorizadas las competencias, se definieron las subcompetencias para cada competencia.

\section{RESULTADOS}

Las competencias que a juicio de las 16 nutricionistas entrevistadas debieran tener en la atención dietoterapéutica del sobrepeso y la obesidad infanto juvenil, se presentan en la tabla 1. Se puede observar que la competencia "realizar diagnóstico integral del estado nutricional", fue valorada como muy importante por las 16 entrevistadas, de igual forma que el concepto "siempre la utilizo". En tanto que sólo 5 de ellas, declaró que "el pregrado contribuyó totalmente a lograr esta competencia". Respecto a la competencia "brindar atención dietoterapéutica a la población infanto

\section{TABLA 1}

Competencias declaradas por nutricionistas desde su puesto de trabajo, para el manejo dietoterapéutico del sobrepeso y de la obesidad en población infanto juvenil.

\section{Competencia}

1. Realizar un diagnóstico integral del estado nutricional: antropométrico, clínico, físico, alimentario y psicosocial.

2. Brindar atención dietoterapéutica al niño y adolescente con sobrepeso y obesidad, con principios éticos.

3. Realizar educación alimentaria a niños y adolescentes sobrepeso y obesidad, familia y comunidad.

4. Participar en diferentes actividades de trabajo comunitario para la prevención del sobrepeso y la obesidad infanto juvenil.

5. Participar en la programación de acciones de prevención de la obesidad infanto juvenil.

6. Integrar el equipo de salud intersectorial e interdisciplinario de prevención del sobrepeso y la obesidad infanto juvenil.

7. Planificar minutas para la prevención, tratamiento, rehabilitación del sobrepeso y la obesidad en población infanto juvenil.

8. Aplicar técnicas de comunicación oral y escrita entre el niño, adolescente obeso y el nutricionista.

9. Revisión periódica de patrones de referencias bibliográficas actualizados para clasificar a los niños y adolescentes en sobrepeso y obesidad.

10. Aplicar encuestas de consumo y de hábitos alimentarios en población infanto juvenil con sobrepeso y obesidad.

$\begin{array}{ccc}\begin{array}{c}\text { Muy } \\ \text { importante }\end{array} & \begin{array}{c}\text { Siempre } \\ \text { la utilizo }\end{array} & \begin{array}{c}\text { El pregrado } \\ \text { contribuyó } \\ \text { a su logro }\end{array}\end{array}$

16

10

13

2

2

2

6

4

0

8
16

9

3

2

0
1

0

0

4

5

0

2 
juvenil con sobrepeso y obesidad, con principios éticos" sólo 10 nutricionistas la declararon como muy importante, 9 declararon utilizarla siempre y ninguna señaló que el pregrado contribuyó totalmente a lograr esa competencia. La competencia referida a "educación alimentaria" fue valorada como muy importante por 13 entrevistadas y 12 de ellas declararon que la utilizaban siempre, en tanto que sólo 2 señalaron que el pregrado contribuyó a su logro. La competencia "Revisión periódica de patrones de referencia bibliográfica actualizados para clasificar a niños y adolescentes en sobrepeso y obesidad" no fue considerada como muy importante por ninguna de las nutricionistas, quienes además declararon no utilizarla.

Del análisis y jerarquización anterior, se obtuvieron 5 competencias específicas y 13 subcompetencias del nutricionista para la atención dietoterapéutica a la población infanto juvenil con sobrepeso y obesidad, las que se muestran en la tabla 2. Se puede observar que la competencia "brindar atención dietética o dietoterapéutica al niño y adolescente con sobrepeso y obesidad, en diversos contextos", aparece con la mayor cantidad de subcompetencias.

La tabla 3 muestra las 3 competencias generales y sus
6 subcompetencias, correspondiendo a la mayoría de las expresadas como "muy importantes" por la nutricionistas entrevistadas.

\section{DISCUSIÓN}

En la actualidad los estudios sobre competencias profesionales y en particular del nutricionista, son escasos, aunque ya en el año 1990, la Organización Panamericana de la Salud, OPS, en su Manual Metodológico para Estudio de los Perfiles Ocupacional y Académico del Nutricionista-Dietista en los países de América Latina, planteaba la necesidad de mejorar la formación académica, el desempeño y la satisfacción en el ejercicio profesional. La respuesta a este desafío se planteaba a través de la estructuración de un currículo basado en competencias (11).

Algunos estudios recientes sobre la formación de Nutricionistas, desde el punto de vista curricular, hacen referencia a insumos básicos para su elaboración, partiendo por la definición de perfil profesional u ocupacional, de perfil de egreso, de las competencias según patologías específicas y ámbitos de acción (8,13-22). En particular las competencias identificadas para la atención dietética y nutricional de la población infanto

\section{TABLA 2}

Competencias específicas y subcompetencias de nutricionistas obtenidas, para la atención dietoterapéutica a la población infanto juvenil con sobrepeso y obesidad.

\section{Competencias específicas}

1. Valorar el estado nutricional de manera integral de población infanto-juvenil con sobrepeso y obesidad.

\section{Brindar atención dietética o} dietoterapéutica al niño y adolescente con sobrepeso y obesidad, en diversos contextos.

3. Educar en dietética y alimentación a niños y adolescentes con sobrepeso y obesidad y a su familia.

4. Participar en actividades comunitarias para el manejo integral del sobrepeso y la obesidad infanto juvenil.

5. Participar en la programación de las actividades de salud del niño adolescente para el manejo integral del sobrepeso y la obesidad infanto juvenil.

\section{Subcompetencias}

1.1 Evaluar el estado nutricional por los diferentes métodos.

1.2 Revisar patrones de referencias bibliográficas actualizados, para clasificar a los niños y adolescentes en sobrepeso y obesidad.

1.3 Establecer las relaciones y participación de cada uno de los métodos en la obtención del diagnostico nutricional integrado.

2.1 Diagnosticar el estado nutricional, con énfasis en el método dietario.

2.2 Aplicar los principios nutricionales en la determinación de los requerimientos de energía, nutrientes y no nutrientes, interpretando sus resultados.

2.3 Formular la prescripción dietética para el caso índice y su familia considerando los antecedentes anteriores.

2.4 Planificar minutas para el manejo integral del sobrepeso y obesidad infanto juvenil.

2.5 Lograr adherencia al tratamiento dietético con el apoyo del equipo de salud.

3.1 Desarrollar una intervención educativa con enfoque sistémico para lograr aprendizajes significativos en conductas de alimentación y nutrición de población infanto juvenil con sobrepeso y obesidad, evaluando su impacto.

4.1 Ejecutar en conjunto con el equipo de salud, actividades de trabajo comunitario del nutricionista, para la prevención de la obesidad infanto juvenil.

4.2 Formular proyectos de intervención alimentario nutricional en población infanto juvenil con sobrepeso y obesidad.

5.1 Programar las actividades del nutricionista en el programa de salud del niño y adolescente en los diferentes niveles y tipos de atención y normativas vigentes y su evaluación.

5.2 Evaluar la programación local del año anterior al momento de programar estas actividades. 
juvenil afectada por el sobrepeso u obesidad, resultantes de este trabajo, permiten contar con información de utilidad para ser incorporadas en los procesos de innovación curricular del pregrado y dar respuesta al actual perfil epidemiológico y nutricional de la población infanto juvenil. En efecto el sobrepeso y la obesidad como problema de salud pública deben ser tratados desde la más temprana edad con profesionales competentes. Los resultados de este estudio pudieran estar limitados por tratarse de una consulta a solo dieciséis Nutricionistas de nivel local y por lo tanto ser extrapolados solo a esta realidad. Bajo esta consideración, Ilama la atención que de las dieciséis nutricionistas entrevistadas, sólo cinco declararan que su formación de pregrado contribuyó totalmente a la adquisición de la competencia "realizar diagnóstico integral del estado nutricional".

Esto pudiera significar una insuficiente capacidad en la aplicación de métodos, instrumentos, equipos y técnicas necesarias para realizar un diagnóstico nutricional correcto.

Más preocupante aún, es que la totalidad de ellas declaró que no fueron formadas para "brindar la atención dietoterapéutica" requerida en estos casos, aunque diez de ellas la consideró "muy importante" y nueve declararon que la utilizaban siempre. Siendo esta competencia esencial y fundamental en la formación de nutricionistas, más allá de su explicitación o no en el currículo, estas respuestas no se condicen con el quehacer permanente de las entrevistadas, quienes a diario deben cumplir esta función. Tal vez esta sería la razón por la que las entrevistadas consideraron que debería ser explicitada en el perfil de egreso.

La competencia referida a "educación alimentaria" fue valorada como muy importante por 13 entrevistadas y 12 de ellas declararon que la utilizaban siempre, sin embargo sólo 2 señalaron que el pregrado contribuyó a su logro, lo que pudiera interpretarse como falta de contenidos educativos específicos para realizar educación alimentaria individual y familiar dentro de los planes de estudio. Al mismo tiempo estos resultados denotan la necesidad de perfeccionamiento y educación continua en estas áreas. Respecto de la competencia "revisión de patrones de referencia actualizados", llama la atención que siendo una competencia formulada por las entrevistadas, ninguna de ellas la consideró muy importante, no siempre la utilizaban y todas declararan no haber recibido formación en el pregrado. Tal vez esta situación podría explicarse por las sucesivas actualizaciones de las normativas ministeriales referentes a esta competencia, las que obligan a los profesionales a cautelar su aplicación como criterio oficial de las actividades que dicen relación con el sobrepeso y la obesidad $(5,6)$. Además, las respuestas expresadas podrían significar un posible sesgo de error en la clasificación del estado nutricional con el consiguiente error de diagnóstico lo que representaría una gran limitación por parte de los profesionales, que se supone deberían tener los conocimientos, las habilidades, actitudes y valores para abordar esta problemática. Lo anterior constituye una señal de alerta para corregir estas deficiencias en el currículo, incorporándolo en los programas de asignaturas, en los resultados de aprendizajes, contenidos educativos, metodologías y estrategias de aprendizajes, en los sistemas de evaluación y monitoreo, y en los niveles de profundidad a lo largo del proceso formativo y su integración con las competencias generales $(14,19)$.

Respecto de las competencias específicas agrupadas de forma lógica y secuencial dentro de la formación profesional y de la atención propiamente tal, fue posible obtener las respectivas subcompetencias que describen en detalle los aspectos parciales a considerar en cada competencia (23). De esta manera las subcompetencias del nutricionista para la atención dietoterapéutica a la población infanto juvenil con sobrepeso y obesidad, son mayores debido a la complejidad de la competencia. El detalle de las subcompetencias podría contribuir a un mejor diagnóstico del estado nutricional; a una determinación más precisa de los requerimientos de energía, nutrientes y no nutrientes y su interpretación; a una mejor formulación y evolución de la prescripción dietética; a una adecuada planificación de minutas y a lograr adherencia al tratamiento dietético con el apoyo del equipo de salud, entre otros (24).

En la competencia "educar en dietética y alimentación

\section{TABLA 3}

Competencias generales y subcompetencias de nutricionistas obtenidas, para la atención dietoterapéutica a la población infanto juvenil con sobrepeso y obesidad.

\section{Competencias generales}

6. Integrar el equipo de salud intersectorial e interdisciplinario para el manejo integral del sobrepeso y obesidad infanto juvenil.

7. Aplicar técnicas de comunicación interpersonal efectiva oral y escrita en el niño, adolescente con sobrepeso y obesidad y su familia.

8. Aplicar los principios éticos profesionales en todas las instancias de acción del manejo integral del sobrepeso y obesidad infanto juvenil.

\section{Subcompetencias}

6.1 Participar en reuniones clínicas, argumentando el plan de intervención dietética y nutricional de casos clínicos con sobrepeso y obesidad infanto juvenil.

6.2 Aplicar técnicas de trabajo grupal y de gestión de trabajo intersectorial e interdisciplinario, en alimentación y nutrición.

7.1 Aplicar los elementos de la comunicación empática, etapas del cambio, técnicas de persuasión y de acogida como parte de la atención dietética o dietoterapéutica ya sea a nivel individual y/o grupal. 7.2 Registrar en el lenguaje técnico las acciones realizadas en los registros establecidos.

8.1 Aplicar los principios éticos al momento de brindar atención dietoterapéutica en diversos contextos.

8.2 Aplicar los principios éticos al momento de registrar, interpretar e informar, el estado nutricional. 
a niños y adolescentes con sobrepeso y obesidad y a su familia.", la sub competencia implica partir por la elaboración de un diagnóstico educativo individual considerando las necesidades educativas, los conocimientos en alimentación y nutrición, los hábitos alimentarios, los resultados de aprendizajes, la selección de los materiales educativos, las estrategias de enseñanza y aprendizaje y la valoración de los mismos aprendizajes, con los resultados de aprendizaje planteados. Lo anterior le permitiría al estudiante adquirir las herramientas para mejorar las conductas alimentarias y modificar la realidad (25 - 27). La participación en actividades comunitarias y en la programación de actividades en salud, correspondientes a competencias específicas, pueden ser logradas a través de las subcompetencias señaladas en la tabla 2 y están presentes en la mayoría de los planes de estudios a nivel nacional e internacional $(17,18,28)$, lo que sustenta la adecuada orientación que deben seguir los nuevos planes de estudio dirigidos a la formación de nutricionistas.

Las tres competencias genéricas y las respectivas subcompetencias, presentadas en la tabla 3, fueron consideradas fundamentales por los analistas, para lograr adherencia al tratamiento dietoterapéutico del sobrepeso y la obesidad infanto juvenil, ya que a través del trabajo en equipo, la comunicación efectiva con el niño y su familia y la aplicación de los principios éticos en todos los momentos y escenarios de la atención, es posible aumentar las probabilidades de éxito en el tratamiento de estas patologías. A nivel general los expertos señalan que los profesionales más exitosos son aquellos que poseen atributos mayormente relacionados con las competencias genéricas, que con las específicas de su profesión $(10,22,29)$, de forma que en la actualidad están siendo consideradas en todos los modelos educativos institucionales y plasmados en determinados programas de asignaturas obligatorias, complementarias e integradoras.

\section{CONCLUSIÓN}

Se concluye que con la metodología aplicada en este trabajo fue posible identificar las competencias y las subcompetencias requeridas por los nutricionistas, para brindar la atención dietoterapéutica a población infanto juvenil con sobrepeso y la obesidad. La información generada, podría ser de utilidad para la formulación y rediseño de planes de estudios destinados a la formación de nutricionistas, y al perfeccionamiento continuo.

\section{RESUMEN}

El objetivo de presente estudio fue identificar las competencias y las subcompetencias requeridas para brindar la atención dietoterapéutica a la población infanto juvenil con sobrepeso y obesidad, que se deben desarrollar en la formación de las nutricionistas. Para identificar las competencias se contó con la asesoría de una experta en el tema. Se aplicó una encuesta semi estructurada por técnica de entrevista personal, a 16 nutricionistas de diversos campos laborales que tenían a su cargo la atención dietoterapéutica de la población infanto juvenil con sobrepeso y obesidad. Con la información obtenida se elaboró un listado de competencias, las que fueron analizadas y categorizadas por un grupo de académicos con el fin de definir las respectivas subcompetencias. Se obtuvo un listado con 5 competencias específicas y 3 competencias generales, con sus respectivas subcompetencias. Se concluye que es posible identificar competencias y subcompetencias requeridos por nutricionistas para brindar la atención dietoterapéutica en población infanto juvenil con sobrepeso y/o obesidad.

Palabras clave: Competencias, subcompetencias, formación de nutricionistas, sobrepeso y obesidad infanto juvenil.

Agradecimientos: Este Trabajo fue realizado en el marco del Proyecto MECESUP UCO 0609. Modelo Educativo Transdisciplinario para la Prevención de la Obesidad Infanto Juvenil. Universidad de Concepción. Unidad de Coordinación institucional. 2007-2010.

\section{BIBLIOGRAFÍA}

1. Ministerio de Salud de Chile, Encuesta Nacional de Salud, ENS, 2009- 2010.

2. Encuesta Nacional de Consumo Alimentario. Informe final, ENCA, 2010-2011. Facultad de Medicina, Universidad de Chile, Santiago, 2014.

3. Ministerio de Salud de Chile, Departamento de promoción de la salud y participación ciudadana. Programa promoción de la Salud. 2012. Disponible en http://www.minsal.cl

4. Ministerio de Salud. Estrategia global contra la obesidad (EGO-Chile). Santiago, 2008. Disponible en http://www. ego-chile.cl

5. Ministerio de Salud de Chile. Norma Técnica Programa Nacional de Salud de la Infancia. 2012.

6. Ministerio de Salud de Chile. Protección de Salud. Programas de Salud del Adolescente. Orientaciones técnicas para el control de salud integral de adolescentes. Año 2014.

7. Tuning Educational Structures In Europe. Informe Final Fase Uno. Universidad de Deusto. España, Sócrates, p. 280. 2003.

8. Colegio de Nutricionistas de Chile. Taller metodológico para definir el perfil ocupacional por competencias, del profesional Nutricionista, año 2006.

9. Centro interuniversitario de desarrollo- CINDA. Grupo operativo de universidades Chilenas, fondo de desarrollo institucional- Mineduc- Chile. Diseño Curricular basado en competencias y aseguramiento de la Calidad en la Educación Superior. Editorial: Alfabeta Artes Gráficas. Santiago, Chile.2009.

10. Centro interuniversitario de desarrollo- CINDA. Grupo operativo de universidades Chilenas, fondo de desarrollo institucional- Mineduc- Chile. Seguimiento de egresados e inserción laboral: Experiencias Universitarias. Editorial: Alfabeta Artes Gráficas. Santiago, Chile. 2012.

11. Organización Panamericana de la Salud, OPS, OMS. Manual Metodológico para Estudio de los Perfiles Ocupacional y Académico del Nutricionista-Dietista en los países de América Latina.1989, Washington, D.C.

12. Bolseguí M, Fuguet Smith A. Construcción de un modelo conceptual a través de la investigación cualitativa. Sapiens. Revista Universitaria de Investigación 20067207229. Disponible en: http://www.redalyc.org/articulo. oa?id=41070114. Fecha de consulta: 30 de diciembre de 2014.

13. Kennedy D. Redactar y Utilizar Resultados de aprendizaje. Un Manual Práctico. Editorial:University College Cork, Iralnda, 2007.

14. Labraña T, AM. Duran F, E. Soto A .Competencias del Nutricionista en el ámbito de atención primaria de salud. Rev ChilNutr. 2005. 32(3):239-46.

15. Fernández, E. Gattas, V. Jara, MJ. Pérez, Y. Pye, C. Competencias del Profesional Nutricionista en la Atención del Paciente Obeso. Rev Colegio Nutricionistas Chile. Santiago, año 2006. 
16. Giacometto C,M. Gómez $M, K$. Opazo M, MA. Razeto $W$, MAE Competencias del Profesional Nutricionista en la Atención del Paciente Renal. Revista Colegio de Nutricionistas de Chile. Santiago, año 2006.

17. Labraña T Ana María, Durán F Eliana, Asenjo I Gisela, Hansel R Gunhild. Plan de estudios basado en competencias para la carrera de nutrición y dietética de la universidad de concepción. Rev Chil Nutr. 2010; 37( 3 ): 302-7.

18. Gonzalez MA, et all. Innovación Curricular por Competencias en la Formación del Nutricionista. Proyecto Mecesup UBB 0606. Editoral Trama Impresores S.A, Enero 2012.

19. López D., Romo ME. Enseñanza de la Dietética por competencias. Rev Col Nutr Chil. 2006; 3:16-7.

20. Schmal $S, R$. Ruiz-Tagle A, A. Una metodología para el diseño de un currículo orientado a las competencias. Rev Chil Ingen, 2008; 16 (1), pp. 147-58.

21. Universidad de Concepción, Proyecto Mecesup, UCO 0609. Informe técnico "Análisis y formulación de Competencias para la atención de personas obesas Infanto-Juvenil. Delia Soto, Concepción, 2009.

22. Coronel S, Díaz R, Ramírez V, Berrún N, et all. Los Nutriólogos en México. Un estudio del mercado laboral. Editorial: Trillas. México. D.F. 2008.

23. Hawes G, Corvalan O. Construcción de un perfil profesional. Proyecto Mecesup Tal 0101, Documento de trabajo
1/2004. Instituto de investigación y desarrollo educacional. Universidad de Talca. Talca Enero 2005.

24. Cornejo V, Cruchet S. Nutrición en el ciclo vital. Ed Mediterraneo Ltda. Santiago-Buenos Aires. 2014.

25. Kaluf, F. C. Reflexiones sobre competencias y educación, en CINDA: Competencias de egresados universitarios. Santiago de Chile. 2004. Recuperado de: http://aula.virtual.ucv.cl/ 30 diciembre, 2014.

26. Olivares S. Educación en nutrición para la acción. Conceptos básicos de Marqueting social en salud Pública en: Nutrición en el ciclo vital. Ed. Mediterraneo Ltda. Santiago-Buenos Aires. 2014, capitulo 18, p 417-30.

27. FAO/Iniciativa América Latina y Caribe sin Hambre. La información, comunicación y educación en alimentación y nutrición para promover la seguridad alimentaria y nutricional en los países de América Latina y el Caribe. Roma: FAO; 2012.

28. González, M. Innovación curricular en Nutrición y Dietética. Un desafío para Chile y América Latina. Ediciones: Universidad del Bío-Bío. Año 2012. ISBN 978-956-781383-4.

29. Modelo de Enseñanza de Competencias Genéricas. Programa de Estudios sobre la Responsabilidad Social. Programa Competencias Genéricas, Convenio de Desempeño UCO 1204. Universidad de Concepción, Mayo 2013. 